\title{
Esfincterotomia Lateral Interna Associada à Hemorroidectomia no Tratamento da Doença Hemorroidária. Vantagem ou Desvantagem?
}

\author{
Open Haemorrhoidectomy with Associated Lateral Internal Sphincterotomy for \\ Treatment of Haemorrhoids: Advantage or Disadvantage?
}

\author{
${ }^{3}$ HÉLIO MOREIRAJUNIOR; ${ }^{3}$ JOSÉPAULOTEIXEIRAMOREIRA; ${ }^{1}$ HÉLIO MOREIRA; \\ ${ }^{4}$ CINTHIA SATOMI IGUMA; ${ }^{2}$ ARMINDACAETANO ALMEIDA $;{ }^{3}$ CRISTIANO NUNES DE MAGALHÃES
}

\author{
${ }^{1}$ Prof. Titular/Chefe do Serv. Coloproctologia - Fac. Medicina U. F. Goiás; ${ }^{2}$ Prof. Serv. Coloproctologia - Fac. Medicina \\ U. F. Goiás; ${ }^{3}$ Membros do staff Serviço de Coloproctologia - Fac..Medicina UF Goiás; ${ }^{4}$ Ex-residente do Serviço de \\ Coloproctologia - Fac. Medicina. UF Goiás.
}

\begin{abstract}
MOREIRA JUNIOR H; MOREIRA JPT; IGUMA CS; ALMEIDA AC; MAGALHÃES CN. Esfincterotomia lateral interna associada à hemorroidectomia no tratamento da doença hemorroidária. Vantagem ou desvantagem?. Rev bras Coloproct, 2007;27(3): 293-303.

RESUMO: Racional: A importância de realizar-se esfincterotomia concomitantemente com hemorroidectomia, para melhor controle de dor pós-operatória, ainda é motivo de grande discussão acadêmica. Objetivos: Estudar as implicações clínicas da esfincterotomia lateral interna associada à hemorroidectomia, no tratamento cirúrgico da doença hemorroidária. Pacientes e Métodos: Foram avaliados 20 pacientes portadores de doença hemorroidária, submetidos à "hemorroidectomia aberta" pela técnica de Miligan-Morgan, distribuídos em dois grupos: Grupo 1: Hemorroidectomia sem esfincterotomia (sem ELI) e Grupo 2: Hemorroidectomia com esfincterotomia (com ELI). Analisou-se a dor e a continência anal pós-operatória utilizando-se parâmetros clínicos e manométricos. A dor, complicações pós-operatórias e a presença de sintomas de incontinência anal foram avaliadas no pós-operatório. Todos os pacientes foram submetidos à eletromanometria anorretal, tanto no pré como no pós-operatório, e os dados coletados foram comparados entre os dois grupos de estudo. Resultados: Não houve diferença, entre os dois grupos, na incidência de complicações pós-operatórias. O uso de narcóticos foi maior no Grupo I nas $1^{\text {as }} \mathbf{2 4}$ horas. Entretanto, a dor foi maior no Grupo II no $3^{\circ}$ e $7^{\circ}$ dia de pós-operatório. O tempo de cicatrização da ferida operatória foi semelhante nos dois grupos. A incidência de sintomas de incontinência anal foi significativamente maior para o grupo tratado com esfincterotomia. Conclusão: A esfincterotomia lateral interna associada à hemorroidectomia para o tratamento de doença hemorroidária avançada não reduziu a dor pós-operatória, além de ter aumentado o risco de incontinência anal.
\end{abstract}

Descritores: Doença hemorroidária, Hemorroidectomia, Esfincterotomia, Eletromanometria.

\section{INTRODUCÃO}

Ao longo dos anos sempre houve muita polêmica em torno do termo "hemorróidas", que geralmente é usado no plural e no sentido de indicar afecção hemorroidária sintomática.

Após a publicação do trabalho de Thomson ${ }^{1}$, tem sido cada vez mais aceito que as hemorróidas são verdadeiros coxins de tecido conectivo fibroelástico, ricos em plexos vasculares e com múltiplas anastomoses arteriovenosas, situados na submucosa da região anorretal, sendo considerados elementos importantes no mecanismo da continência anal.

Do ponto de vista etiopatogênico, o reconhecimento anatômico desses coxins reforça a teoria do deslizamento do assoalho anal ${ }^{1,2}$ e explica, de maneira satisfatória, a evolução das hemorróidas ao longo das faixas etárias, com o aparecimento dos sintomas em virtude da perda da elasticidade e da rotura dos elementos estruturais de sustentação

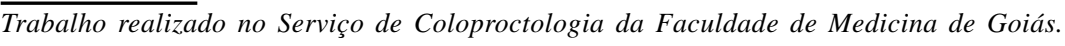

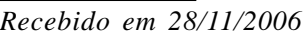

Aceito para publicação em 25/01/2007 
Devido ao fato do tecido hemorroidário estar presente desde o nascimento do indivíduo, quando as estruturas estão intactas, considera-se patológico apenas hemorróidas que produzem sintomas. Para efeito de conceituação, utilizaremos à designação "doença hemorroidária" para indicar apenas os casos sintomáticos.

A doença hemorroidária é uma afecção bastante comum nos consultórios de coloproctologia e o seu tratamento, dependendo do quadro evolutivo, recai em duas modalidades principais: não cirúrgicas e cirúrgicas. Em muitos pacientes, os sintomas podem ser tratados e muitas vezes são realmente aliviados utilizando-se de medidas simples como a orientação para melhor higiene local, melhor hábito dietético e prevenção do esforço evacuatório excessivo com correção do hábito intestinal.

A hemorroidectomia, quando bem indicada e tecnicamente bem conduzida, é a modalidade de tratamento que traz melhores resultados terapêuticos para a doença hemorroidária e deverá ser sempre considerada nos seguintes casos:

- pacientes que não respondem às repetidas tentativas de tratamento clínico;

- hemorróidas prolapsadas e que exijam freqüentes reduções manuais;

. hemorróidas complicadas por estrangulamento, trombose ou alguma outra lesão associada, como a fissura anal, por exemplo.

Com o progresso tecnológico da medicina, novos equipamentos vêm sendo incorporados à prática médica e o uso de alguns deles permite estudar, cada vez mais detalhadamente, a fisiologia do cólon, reto e do ânus. Um destes métodos é a eletromanometria anorretal, capaz de fornecer valiosas informações sobre a fisiologia anorretal e do assoalho pélvico. Este exame auxilia os estudos das pressões esfincterianas, da sensibilidade, da capacidade e da complacência retal e do reflexo inibitório retoanal. A determinação das pressões anais de forma mais precisa pode levar a decisões terapêuticas mais objetivas ${ }^{3}$. Além disso, a avaliação eletromanométrica pode, também, ser usada como um padrão, para comparação de resultados funcionais depois de determinado tratamento ${ }^{3,4}$.

Vários estudos de eletromanometria anorretal demonstraram que, pacientes portadores de doença hemorroidária, podem apresentar pressão do canal anal aumentada, semelhantemente ao que ocorre na fissura anal ${ }^{4,5,7,8}$. Existem vários relatos na literatura que mos- tram que a hipertonia do esfíncter interno do ânus exerce um papel etiológico, tanto no desenvolvimento da fissura anal, como na doença hemorroidária ${ }^{6,9}$.

Alguns autores sugerem que a esfincterotomia lateral interna associada à hemorroidectomia, pode contribuir para uma melhor evolução clínica no pósoperatório (redução da dor, da retenção urinária, da estenose anal e do tempo de cicatrização da ferida

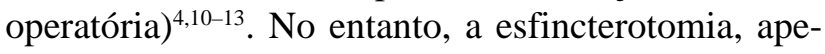
sar de ter seu valor comprovado no tratamento de fissuras anais crônicas, mostrando bons resultados no relaxamento do esfíncter interno do ânus e conseqüente cicatrização da ferida fissurária, provoca alterações esfincterianas irreversíveis, permanentes, podendo mesmo causar algum grau de incontinência fecal ${ }^{14,15}$.

Baseados nestas evidências consideramos oportuno e principalmente importante a avaliação, tanto clínica como fisiológica (manometria anorretal), de pacientes submetidos à hemorroidectomia acrescida de esfincterotomia lateral, no sentido de trazer novos subsídios para o tratamento da doença hemorroidária.

\section{OBJETIVOS}

Os objetivos desse estudo foram o de determinar se a esfincterotomia lateral interna associada à hemorroidectomia, no tratamento cirúrgico da doença hemorroidária, contribui para a redução da dor no pósoperatório (PO), assim como para a diminuição do tempo de cicatrização, da possível retenção urinária e da estenose anal, além de avaliar clinicamente e manometricamente a incidência de incontinência fecal, e comparar estes resultados antes e após o tratamento cirúrgico.

\section{PACIENTES E MÉTODOS}

Este estudo foi realizado no Serviço de Coloproctologia do Departamento de Cirurgia da Faculdade de Medicina da Universidade Federal de Goiás, coletando-se os dados de um banco computadorizado que armazena informações dos pacientes tratados pelo Serviço, de forma prospectiva, durante o período de Maio de 2001 a Junho de 2002.

Foram identificados 20 pacientes portadores de doença hemorroidária, com indicação de tratamento cirúrgico, e que preenchiam os seguintes critérios de inclusão:

1) Idade até no máximo 45 anos; 
2) Doença hemorroidária interna, externa ou mista não complicada (trombose, pseudo-estrangulamento), com indicação de tratamento cirúrgico;

3) Ausência de doenças orificiais associadas;

4) Ausência de cirurgias orificiais prévias ou antecedentes de trauma no local;

5) Ausência de queixas clínicas de incontinência fecal no pré-operatório.

6) Manometria anorretal no pré e pós - operatório.

Esses pacientes formaram dois grupos de estudo, sendo a inclusão em cada grupo definida de forma aleatória, de acordo com a preferência do cirurgião que operou cada caso:

Grupo 1: Hemorroidectomia sem esfincterotomia

Grupo 2: Hemorroidectomia com esfincterotomia

Todos os pacientes foram submetidos à hemorroidectomia pela técnica aberta, obedecendo aos detalhes preconizados por Milligan-Morgan, associada ou não a esfincterotomia lateral interna. Os pacientes selecionados foram submetidos à anestesia raquimedular e restrição de liquido endovenoso durante o procedimento cirúrgico para que não houvesse interferência da hidratação na avaliação de possível retenção urinária no pós-operatório. Não foi realizado antibioticoterapia em nenhum destes pacientes estudados. Não foi utilizado eletrocautério e nem foram feitas infiltrações anestésicas locais em nenhum dos pacientes. A esfincterotomia, quando realizada, foi feita na porção lateral esquerda do esfíncter interno do ânus, com secção de aproximadamente $1 / 3$ da sua extensão. Todos os pacientes permaneceram internados pelo período de 24 horas após a realização do procedimento cirúrgico para melhor avaliação da dor.

A analgesia e cuidados gerais no pós-operatório foram padronizados da seguinte maneira:

1) Diclofenaco sódico $50 \mathrm{mg} \mathrm{VO}$ de $8 / 8$ horas por três dias,

2) Dipirona sódica 500mg VO até a cada $6 / 6$ horas, se necessário, de acordo com a intensidade da dor;

2) Meperidina - dose de $0,5 \mathrm{mg} / \mathrm{kg}$ IM quando necessário, em caso de dor não controlada pelos analgésicos anteriores, nas primeiras 24 horas.

3) Pomada à base de substância heparinóide, sem corticóide, recobrindo a ferida operatória - duas a quatro vezes ao dia,

4) Banhos de assento com água morna de três a seis vezes ao dia;
5) Orientação básica dietética rica em fibras e ingestão adequada de líquidos.

Na ocasião da alta hospitalar, todos os pacientes recebiam um formulário contendo uma escala visual analógica, para avaliação da dor nos primeiros sete dias após a cirurgia. Essa escala graduava a dor em três intensidades: sem ou pouca dor, dor moderada e dor intensa. Foi solicitado aos pacientes o preenchimento do formulário, em domicílio, conforme a intensidade da dor no decorrer de cada um dos sete dias.

A dor foi avaliada de três formas:

1) Nas primeiras 24 horas, utilizando-se com parâmetro a necessidade do uso de analgésicos opiáceos;

2) Do $1^{\circ}$ ao $7^{\circ}$ dia pós-operatório, utilizando-se escala analógica visual e a necessidade do uso de analgésicos;

3) De maneira global, nos 45 dias de acompanhamento ambulatorial, pelas informações verbais do paciente.

Incontinência fecal foi avaliada, clinicamente, no pré e pós-operatório, utilizando-se de um questionário preconizado por outros autores ${ }^{16}$ através do qual obtem-se um valor numérico para o grau de continência, variando de 28 a 128, correspondendo, respectivamente, à incontinência total e a continência perfeita.

Os pacientes inclusos neste estudo foram revistos, no ambulatório, seis meses após a cirurgia, para reavaliação clínica final da continência fecal.

Os exames manométricos foram feitos em um único aparelho e obedeceram à mesma técnica já descrita e padronizada, anteriormente por outros autores ${ }^{17}$. Os valores eletromanométricos, considerados como normais neste estudo, também foram motivo de outras publicações na literatura ${ }^{18}$.

\section{Análise Estatística}

Para análise e interpretação estatística de variáveis qualitativas envolvendo dados do mesmo paciente (manometria pré e pós-operatória) foi utilizado o teste de Wilcoxon; para variáveis qualitativas envolvendo dados de pacientes em grupos distintos (Grupo 1 vs. Grupo 2 ; Continente vs. Incontinente) utilizou-se o Teste Exato de Fisher. No caso de variáveis quantitativas de grupos distintos, foram utilizados testes de Médias, tais como Análise de Variância e teste U de Mann-Whitney. Para comparar a intensidade da dor pós-operatória entre os grupos 1 e 2 , foi utilizado o teste U de Mann-Whitnney. 


\section{RESULTADOS}

Foram identificados 20 pacientes que foram aleatoriamente distribuídos nos 2 seguintes grupos:

- Grupo 1 ( $\mathrm{n}=10)$ : hemorroidectomia sem esfincterotomia,

- Grupo 2 ( $\mathrm{n}=10)$ : hemorroidectomia com esfincterotomia.

Os dois grupos foram semelhantes em relação à idade e ao número de mamilos hemorroidários ressecados. Apesar da diferença na proporção de pacientes do sexo feminino e masculino entre o grupo 1 e o grupo 2 , a mesma não foi estatisticamente significante $(\mathrm{p}=0,135) \quad($ Tabela 1$)$.

Nenhum dos 20 pacientes estudados apresentou sangramento, infecção de ferida ou estenose anal no pós-operatório. $\mathrm{O}$ índice de retenção urinária foi o mesmo nos dois grupos (Tabela 2).

A necessidade do uso de narcóticos para analgesia nas primeiras 24 horas de pós-operatório, foi discretamente maior no grupo1 (40\% vs. $30 \%)$. Apenas um paciente de cada grupo necessitou de mais de uma dose desta medicação (Fig. 1).
O uso de dipirona sódica, associado a AINH no pós-operatório, foi necessário em $80 \%$ dos pacientes submetidos à hemorroidectomia sem esfincterotomia e em 70\% dos pacientes submetidos à hemorroidectomia com esfincterotomia, não havendo diferença, estatisticamente significante, entre os dois grupos. (Fig. 2).

A intensidade da dor no pós-operatório, avaliada por meio de uma escala visual analógica, foi menor no grupo sem esfincterotomia. No entanto, essa diferença só foi estatisticamente significante no $3^{\circ}$ e $7^{\circ}$ dia de pós-operatório (Fig. 3).

Em relação ao período de cicatrização da ferida cirúrgica, não houve diferença estatisticamente significante, entre os pacientes do grupo 1 e grupo 2 ( Tabela 3).

A incontinência fecal foi avaliada, clinicamente, 45 dias após a cirurgia, utilizando-se um questionário ${ }^{16}$ respondido pelo próprio paciente, sob supervisão de médico que não participou do ato cirúrgico. Houve diferença, estatisticamente significante, quando se comparou o grupo 1 com o grupo 2 (Tabela 4).

No grupo 1 (sem ELI), 20\% dos pacientes apresentaram incontinência para gases 1-3 vezes/ mês,

Tabela 1 - Dados demográficos dos pacientes submetidos à hemorroidectomia com ou sem esfincterotomia.

\begin{tabular}{|c|c|c|c|c|c|}
\hline & & $\begin{array}{l}\text { Grupo 1 } \\
(n=10)\end{array}$ & & $\begin{array}{l}\text { Grupo 2 } \\
(\mathbf{n}=10)\end{array}$ & $\mathbf{P}$ \\
\hline \multicolumn{6}{|l|}{ Sexo } \\
\hline Feminino & 9 & $(90,0 \%)$ & 6 & $(60,0 \%)$ & \\
\hline Masculino & 1 & $(10,0 \%)$ & 4 & $(40,0 \%)$ & $\mathrm{ns}$ \\
\hline Idade & 33,90 & $\pm 8,77$ & 28,80 & $\pm 7,44$ & $\mathrm{~ns}$ \\
\hline \multicolumn{6}{|c|}{ Mamilos ressecados } \\
\hline 3 (Três) & 6 & $(60,0 \%)$ & 3 & $(30,0 \%)$ & \\
\hline 4 (Quatro) & 4 & $(40,0 \%)$ & 7 & $(70,0 \%)$ & ns \\
\hline
\end{tabular}

Tabela 2 - Complicações pós-operatórias precoces e tardias dos pacientes do Grupo 1 (sem ELI) e grupo 2 (com ELI).

\begin{tabular}{|c|c|c|c|}
\hline & $\begin{array}{l}\text { Grupo } 1 \\
(\mathbf{n}=10)\end{array}$ & $\begin{array}{r}\text { Grupo } 2 \\
(\mathbf{n}=10)\end{array}$ & $\mathbf{P}$ \\
\hline Sem Complicações & $8 \quad(80,0 \%)$ & $8 \quad(80,0 \%)$ & ns \\
\hline Sangramento & $0 \quad(0,0 \%)$ & $0 \quad(0,0 \%)$ & ns \\
\hline Retenção Urinária & $2(20,0 \%)$ & $2(20,0 \%)$ & ns \\
\hline Infecção & $0 \quad(0,0 \%)$ & $0 \quad(0,0 \%)$ & ns \\
\hline Estenose & $(0,0 \%)$ & $(0,0 \%)$ & ns \\
\hline
\end{tabular}



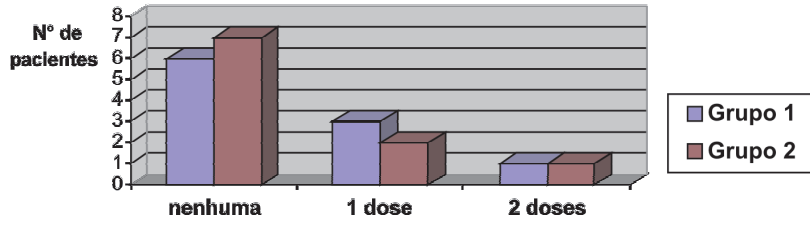

$p>0,05$

Figura 1 - Analgesia, com narcóticos, nas primeiras 24 horas após a cirurgia.

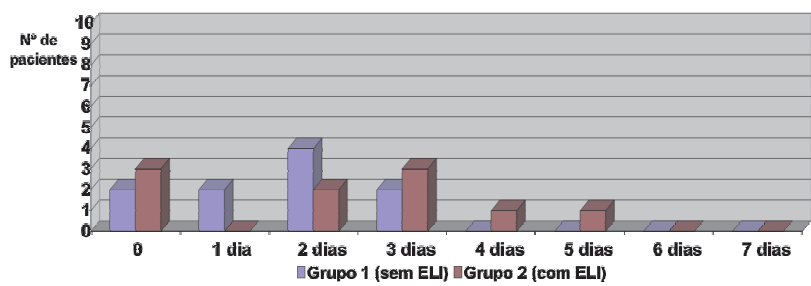

Figura 2 - Tempo de uso da dipirona para analgesia no pósoperatório dos pacientes do grupo 1 (sem ELI) e grupo 2 (com ELI).

porém sem limitação social. No grupo 2 (com ELI), $60 \%$ dos pacientes apresentaram incontinência, sendo: dois para gases (1-3 vezes/ mês), um com incontinência para gases (2-3 vezes/ dia), um com "soiling" ( 2 ou mais vezes/ semana), um com urgência evacuatória para fezes líquidas ( $1 \mathrm{vez} / \mathrm{semana}$ ) e um com incontinência para fezes líquidas (1 vez/ semana).

No entanto, do total de pacientes do grupo 2 (com ELI), com problemas de continência, apenas dois deles referiram discreta limitação social, que não impedia suas atividades habituais (Figura 4).

Seis meses após a cirurgia, apesar de referirem gradativa melhora clínica, 3 pacientes que foram submetidos à hemorroidectomia com esfincterotomia ainda se queixavam de incontinência para gases, 1 vez/ semana, porém, sem limitação social.

Os achados eletromanométricos pré-operatórios não mostraram diferença, estatisticamente significante, entre os dois grupos, em relação à $1^{\text {a }}$ sensação de enchimento retal, volume máximo tolerável

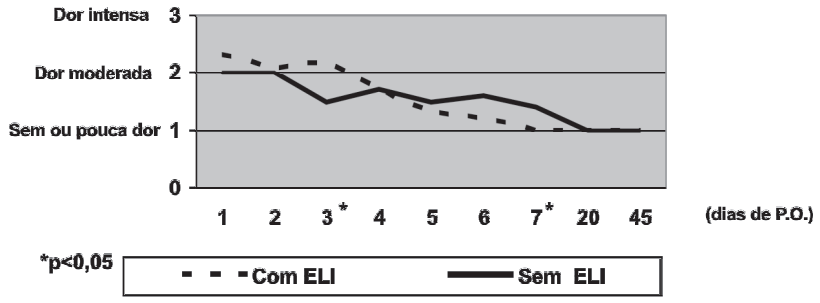

Figura 3 - Intensidade da dor no pós-operatório.

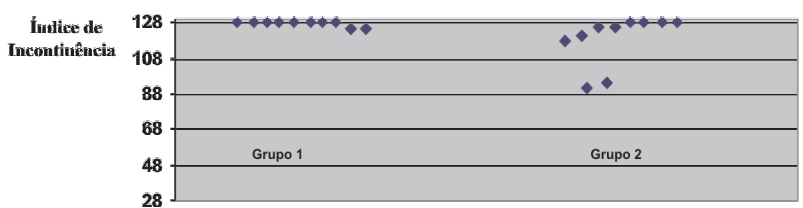

Figura 4 - Gráfico de dispersão dos índices de incontinência dos pacientes do grupo 1 (sem ELI) e do grupo 2 (com ELI). Observe os dois pacientes abaixo do índice 100 de incontinência que apresentavam limitação social.

intra-retal, capacidade retal, pressões de repouso e contração voluntária, zona de maior pressão e a presença do reflexo inibitório retoanal (RIRA).

Comparando os resultados da manometria préoperatória com a manometria pós-operatória nos grupos 1 e 2, observou-se que houve diferença, estatisticamente significante $(\mathrm{p}<0,05)$, para o volume máximo e a capacidade retal nos pacientes submetidos à hemorroidectomia sem esfincterotomia (grupo 1).

Nos pacientes submetidos à hemorroidectomia com esfincterotomia (grupo 2), houve diferença, estatisticamente significante $(\mathrm{p}<0,05)$, entre a pressão de repouso pré-operatória comparada com a pós-operatória (Tabela 5).

Os pacientes do grupo 2, submetidos à hemorroidectomia com esfincterotomia, foram classificados como indivíduos com continência fecal ou incontinentes, baseando-se na pontuação obtida em questionário de incontinência fecal, respondido no $45^{\circ}$ dia após a cirurgia (Tab 4)).

Tabela 3 - Tempo de cicatrização completa da ferida operatória nos pacientes submetidos à hemorroidectomia com e sem esfincterotomia lateral interna.

\begin{tabular}{lccc}
\hline & $\begin{array}{c}\text { Grupo 1 } \\
\text { (sem ELI) }\end{array}$ & $\begin{array}{c}\text { Grupo 2 } \\
\text { (com ELI) }\end{array}$ & P \\
\hline Tempo de Cicatrização (dias) & $32,0 \pm 9,4$ & $28,8 \pm 9,2$ & $\mathrm{~ns}$ \\
\hline
\end{tabular}


Rev bras Coloproct Julho/Setembro, 2007
Esfincterotomia lateral interna associada à hemorroidectomia no tratamento da doença hemorroidária. Vantagem ou desvantagem?

Vol. 27 $\mathbf{N}^{\mathbf{0}} 3$

Tabela 4 - Índice de incontinência fecal dos pacientes submetidos à hemorroidectomia com e sem esfincterotomia.

\begin{tabular}{lccc}
\hline & $\begin{array}{c}\text { Grupo 1 } \\
\text { (sem ELI) }\end{array}$ & $\begin{array}{c}\text { Grupo 2 } \\
\text { (com ELI) }\end{array}$ & P \\
\hline Índice de Incontinência Fecal & $127,8 \pm 0,4$ & $119,5 \pm 15,4$ & $<0,05$ \\
\hline
\end{tabular}

Comparando os resultados da manometria préoperatória entre os pacientes continentes e incontinentes, não houve diferença, estatisticamente significante, em relação à extensão da zona de maior pressão e as pressões de repouso e de contração do canal anal. Estes resultados demonstram a dificuldade de se selecionar qual paciente, com hipertonia esfincteriana no pré-ope- ratório, se beneficiaria da associação de esfincterotomia à hemorroidectomia, uma vez que os achados eletromanométricos entre aqueles que permaneceram continentes e os outros que apresentaram incontinência fecal no pós-operatório são semelhantes.

Comparando os resultados da manometria préoperatória com os da pós-operatória, nesses mesmos

Tabela 5 - Manometria pré e pós-operatória dos pacientes do grupo 1 (sem ELI) e do grupo 2 (com ELI).

\begin{tabular}{|c|c|c|c|}
\hline & $\begin{array}{c}\text { Manometria } \\
\text { pré-operatória }\end{array}$ & $\begin{array}{c}\text { Manometria } \\
\text { pós-operatória }\end{array}$ & $\mathbf{p}$ \\
\hline \multicolumn{4}{|c|}{$1^{\text {a }}$ Sensação retal $(\mathrm{ml})$} \\
\hline Grupo 1 (sem ELI) & $26,0 \pm 20,5$ & $22,0 \pm 6,7$ & ns \\
\hline Grupo 2 (com ELI) & $22,0 \pm 8,9$ & $19,4 \pm 12,4$ & ns \\
\hline \multicolumn{4}{|c|}{ Volume Máximo (ml) } \\
\hline Grupo 1 (sem ELI) & $180,0 \pm 55,8$ & $137,5 \pm 32,8$ & $<0,05$ \\
\hline Grupo 2 (com ELI) & $175,0 \pm 89,1$ & $165,0 \pm 47,6$ & ns \\
\hline \multicolumn{4}{|c|}{ Capacidade Retal (ml) } \\
\hline Grupo 1 (sem ELI) & $154,0 \pm 58,9$ & $115,5 \pm 31,0$ & $<0,05$ \\
\hline Grupo 2 (com ELI) & $153,0 \pm 86,5$ & $145,6 \pm 46,3$ & ns \\
\hline \multicolumn{4}{|c|}{ Pressão de Repouso (mmHg) } \\
\hline Grupo 1 (sem ELI) & $80,8 \pm 16,8$ & $73,5 \pm 25,3$ & ns \\
\hline Grupo 2 (com ELI) & $84,3 \pm 17,3$ & $54,4 \pm 14,1$ & $<0,05$ \\
\hline \multicolumn{4}{|c|}{ Pressão de Contração (mmHg) } \\
\hline Grupo 1 (sem ELI) & $110,0 \pm 50,7$ & $83,7 \pm 27,1$ & ns \\
\hline Grupo 2 (com ELI) & $156,1 \pm 72,3$ & $132,1 \pm 96,2$ & ns \\
\hline \multicolumn{4}{|c|}{ Zona de Maior Pressão (cm) } \\
\hline Grupo 1 (sem ELI) & $2,30 \pm 0,67$ & $2,30 \pm 0,67$ & ns \\
\hline Grupo 2 (com ELI) & $2,20 \pm 0,63$ & $2,11 \pm 0,60$ & $\mathrm{~ns}$ \\
\hline \multicolumn{4}{|c|}{ Reflexo Inibitório Reto Anal } \\
\hline Grupo 1 (sem ELI) & $(+)$ & $(+)$ & ns \\
\hline Grupo 2 (com ELI) & $(+)$ & $(+)$ & ns \\
\hline
\end{tabular}


pacientes (continentes e incontinentes), observou-se que aqueles que não tinham limitação social em decorrência de problemas de continência fecal, apresentaram uma diminuição, estatisticamente significante $(\mathrm{p}<0,05)$, da pressão de repouso após hemorroidectomia com esfincterotomia.

Nos pacientes incontinentes, com limitação social, houve um aumento da pressão de repouso do canal anal, após a cirurgia; no entanto, esse aumento não foi estatisticamente significante (Tabela 6).

Ficou evidente que o encurtamento da zona de maior pressão somente ocorreu nos pacientes sintomáticos, reforçando a teoria de que a deformidade anatômica, provocada pela esfincterotomia, exerce um papel importante no aparecimento dos sintomas de incontinência fecal.

\section{DISCUSSÃO}

A doença hemorroidária é uma afecção bastante comum nos consultórios de coloproctologia e o seu tratamento deve ser direcionado conforme a intensidade dos sintomas e o grau de evolução da doença ${ }^{19}$. A maioria dos pacientes com hemorróidas sintomáticas pode ser tratada, com sucesso, conservadoramente, com a modificação da dieta alimentar, melhora do hábito intestinal e conseqüente prevenção do esforço evacuatório excessivo, além de associar banhos de

Tabela 6 - Resultados da eletromanometria anorretal nos pacientes continentes e incontinentes do grupo 2 (com ELI).

\begin{tabular}{|c|c|c|c|}
\hline & $\begin{array}{c}\text { Manometria } \\
\text { pré-operatória }\end{array}$ & $\begin{array}{c}\text { Manometria } \\
\text { pós-operatória }\end{array}$ & $\mathbf{p}$ \\
\hline \multicolumn{4}{|l|}{$1^{\text {a }}$ Sensação retal $(\mathrm{ml})$} \\
\hline Continentes $(n=8)$ & $20,6 \pm 8,6$ & $15,0 \pm 5,0$ & ns \\
\hline Incontinentes $(\mathrm{n}=2)$ & $27,5 \pm 10,6$ & $35,0 \pm 21,2$ & ns \\
\hline \multicolumn{4}{|l|}{ Volume Máximo (ml) } \\
\hline Continentes $(n=8)$ & $147,5 \pm 37,7$ & $149,2 \pm 34,4$ & ns \\
\hline Incontinentes $(\mathrm{n}=2)$ & $285,0 \pm 176,7$ & $220,0 \pm 56,5$ & ns \\
\hline \multicolumn{4}{|c|}{ Capacidade Retal (ml) } \\
\hline Continentes $(\mathrm{n}=8)$ & $126,8 \pm 42,1$ & $134,2 \pm 34,5$ & ns \\
\hline Incontinentes $(n=2)$ & $257,5 \pm 166,1$ & $185,0 \pm 77,7$ & ns \\
\hline \multicolumn{4}{|c|}{ Pressão de Repouso (mmHg) } \\
\hline Continentes $(n=8)$ & $88,6 \pm 16,6$ & $49,0 \pm 10,3$ & $<0,05$ \\
\hline Incontinentes $(\mathrm{n}=2)$ & $67,0 \pm 1,4$ & $73,5 \pm 3,5$ & ns \\
\hline \multicolumn{4}{|c|}{ Pressão de Contração (mmHg) } \\
\hline Continentes $(n=8)$ & $164,3 \pm 71,3$ & $138,4 \pm 105,8$ & ns \\
\hline Incontinentes $(\mathrm{n}=2)$ & $123,0 \pm 93,3$ & $110,0 \pm 74,9$ & ns \\
\hline \multicolumn{4}{|c|}{ Zona de Maior Pressão (cm) } \\
\hline Continentes $(n=8)$ & $2,12 \pm 0,64$ & $2,28 \pm 0,48$ & ns \\
\hline Incontinentes $(\mathrm{n}=2)$ & $2,50 \pm 0,70$ & $1,50 \pm 0,70$ & ns \\
\hline \multicolumn{4}{|c|}{ Reflexo Inibitório Reto Anal } \\
\hline Continentes $(\mathrm{n}=8)$ & $100 \%$ & $100 \%$ & ns \\
\hline Incontinentes $(\mathrm{n}=2)$ & $100 \%$ & $100 \%$ & ns \\
\hline
\end{tabular}


assento ou pelo menos a higiene anal com água e eventual uso tópico de pomadas.

Se os sintomas persistirem, pode-se optar por outras modalidades de tratamentos, que embora invasivos, são não-cirúrgicos, como: escleroterapia, ligadura elástica e coagulação por raio infravermelho $(\mathrm{CRIV})^{20}$. Alguns estudos têm demonstrado que a ligadura elástica, assim como a coagulação por raio infravermelho (CRIV), têm eficácias semelhantes quanto aos resultados, sendo, no entanto, superiores quando comparadas com a escleroterapia ${ }^{21,22}$. A escolha do tratamento por ligadura elástica ou por CRIV deve ser individualizada, de acordo com a preferência do paciente e a experiência do médico, considerando que a ligadura elástica pode estar associada à dor após o procedimento e a CRIV, além de ter um custo maior, pode necessitar de sessões de tratamento adicionais ${ }^{20}$.

O tratamento cirúrgico deve ser reservado para pacientes que não obtiveram sucesso com o tratamento, dito conservador ou não cirúrgico. Embora a hemorroidectomia apresente melhores resultados terapêuticos a longo prazo, e seja o único procedimento eficaz para tratar hemorróidas externas volumosas, comumente ocasiona dor pós-operatória e está associada a maior risco de complicações, como retenção urinária, sangramento, estenose e infecção.

Várias técnicas cirúrgicas têm sido descritas para o tratamento da doença hemorroidária; as mais realizadas são Milligan-Morgan ${ }^{23}$ (hemorroidectomia aberta) e Ferguson-Heaton ${ }^{24}$ (hemorroidectomia fechada), que apresentam eficácia e segurança semelhantes.

O uso de laser para o tratamento cirúrgico de hemorróidas não oferece vantagens sobre a hemorroidectomia tradicional. Recentemente, tem sido feito o tratamento cirúrgico da doença hemorroidária, utilizando-se um grampeador $(\mathrm{PPH})^{25}$, que permite a ressecção circunferencial do tecido hemorroidário, com reposicionamento e fixação do anoderma em sua localização original ${ }^{26,27}$.

Apesar desse procedimento causar menos dor pós-operatória e um período de recuperação mais curto, ainda não está bem definido a sua eficácia, a longo prazo. Independente da técnica cirúrgica utilizada para o tratamento da doença hemorroidária avançada, a chave para um bom resultado é evitar complicações pós-operatórias.

Dor é a complicação mais freqüente e também a mais temida, causando ansiedade nos pacientes e nos médicos ${ }^{28}$. Desde que a dor após hemorroidectomia, seja adequadamente controlada, torna-se possível a realização desta, de forma ambulatorial, diminuindo os custos do tratamento cirúrgico $^{9,28,29}$.

Referências da literatura têm demonstrado que pacientes portadores de doença hemorroidária, assim como de fissura anal, apresentam pressões anais aumentadas, à custa de hipertonia do esfíncter anal interno ${ }^{5,6,14}$. No entanto, ainda não está bem definido se o aumento da pressão anal é secundário à presença dos coxins vasculares ${ }^{30,31}$, e se interfere no retorno venoso do plexo hemorroidário interno, causando degeneração das estruturas fibromusculares e deslizamento progressivo dos coxins anais ${ }^{32,33}$. Hipertonia do esfíncter anal interno também é descrita como uma possível causa de dor nas cirurgias anorretais, incluindo a hemorroidectomia. Desta forma, a esfincterotomia associada à hemorroidectomia tem sido proposta para reduzir este aumento da pressão anal, podendo, inclusive, diminuir as complicações pós-operatórias (dor, retenção urinária, estenose) $)^{12,34,35}$. Alguns autores consideram que hemorroidectomia isolada normaliza as pressões anais, uma vez que estas estariam aumentadas devido à presença dos coxins vasculares, e que a esfincterotomia, além de ser desnecessária, poderia causar incontinência anal ${ }^{34,36}$.

No presente estudo observou-se que $85 \%$ dos pacientes portadores de doença hemorroidária apresentavam pressões anais aumentadas. Hemorroidectomia associada à esfincterotomia lateral interna (ELI) promoveu uma redução estatisticamente significante $(\mathrm{p}<0,05)$ das pressões de repouso do canal anal, o que não foi observado nos pacientes submetidos à hemorroidectomia sem esfincterotomia. Considerando que a hipertonia do esfíncter anal fosse causada pela presença dos coxins hemorroidários, era de se esperar que as pressões anais retornassem aos valores manométricos normais após ressecção dos mesmos. O fato das pressões anais permanecerem elevadas após hemorroidectomia sem esfincterotomia, como demonstrado neste e em outros estudos ${ }^{12,37}$, sugere que a hipertonia esfincteriana tenha um papel etiológico na formação das hemorróidas.

A hemorroidectomia é considerada uma cirurgia que cursa com variada intensidade de dor no pósoperatório, embora a dor referida pelos pacientes também possa ser influenciada pela sua ansiedade e sensibilidade, dificultando a avaliação da sua intensidade. A 
utilização de uma escala visual analógica, associada à necessidade ou não de analgésicos (meperidina, dipirona) e a quantidade utilizada, parece ser um método de avaliação possível e adequado, conforme demonstrado em alguns estudos anteriores ${ }^{9,35,38}$.

A necessidade do uso de analgésicos no pósoperatório não mostrou diferença, estatisticamente significante, em ambos os grupos. A intensidade da dor, avaliada por uma escala visual analógica, foi discretamente maior nos pacientes submetidos à ELI nos primeiros três dias após a cirurgia, porém, com diferença estatisticamente significante $(\mathrm{p}<0,05)$ somente no $3^{\circ}$ PO. A partir do $4^{\circ}$ dia, os pacientes dos dois grupos evoluíram, simultaneamente, com diminuição progressiva da dor.

Pacientes submetidos à hemorroidectomia com ELI apresentaram menor dor, quando comparados com os pacientes do outro grupo (sem ELI), porém, com diferença, estatisticamente significante, apenas no $7^{\circ}$ PO. Curiosamente, a intensidade da dor pós-operatória nos três primeiros dias (período da $1^{\mathrm{a}}$ evacuação) foi maior nos pacientes do grupo com ELI, sugerindo que o espasmo do esfíncter anal não tem contribuição importante na dor pós-hemorroidectomia ${ }^{34,39}$. Neste estudo, assim como em outros ${ }^{34}$, observa-se que a associação de ELI no tratamento da doença hemorroidária não reduziu a dor pós-operatória.

A incidência de $20 \%$ de retenção urinária, encontrada neste estudo, semelhante ao que já foi relatado por outros autores ${ }^{19,27}$, não teve diferença estatística entre os dois grupos (com e sem ELI). Embora houvesse limitação da quantidade de fluido infundido no peri-operatório, provavelmente a raqui anestesia, assim como a dor perineal, com subseqüente disfunção do trígono vesical ${ }^{19}$, tenham tido alguma participação nestas ocorrências. Esse fenômeno foi demonstrado por Lyngdorf e cols. ${ }^{41}$, que mediu o volume urinário residual e o fluxo urinário (urofluxometria) de pacientes após hemorroidectomia. Nesta sua série de 37 pacientes, os autores observaram que sete pacientes, embora assintomáticos, tinham grande volume residual no pós-operatório. Em relação ao tempo de cicatrização da ferida operatória, taxa de infecção, sangramento e estenose, este estudo não mostrou diferença estatisticamente significante, entre os dois grupos.

Alteração temporária da continência fecal, com ocorrência de "soiling" e urgência fecal após hemorroidectomia foi relatada e atribuída à ressecção dos coxins vasculares ${ }^{31}$. Geralmente, a completa con- tinência anal retorna em alguns meses ${ }^{12,41}$. Alguns autores receiam que a hemorroidectomia associada à ELI aumente o risco de incontinência fecal ${ }^{34,36}$, porém, inúmeros estudos sobre tratamento cirúrgico, com esfincterotomia, da fissura anal mostraram baixa incidência de incontinência anal, quando analisada à longo prazo, sendo, portanto, considerada como de ocorrência transitória ${ }^{42}$.

No presente estudo, dois pacientes do grupo sem ELI e seis pacientes do grupo com ELI apresentaram variados graus de incontinência, principalmente para gases e fezes líquidas, 45 dias após as cirurgias. Dois pacientes, um do sexo masculino e outro feminino, do grupo com ELI, persistiram com incontinência somente para gases, em seis meses de acompanhamento ambulatorial.

Garcia-Aguilar e cols ${ }^{8}$ relataram que a extensão da ELI é considerada um fator de risco para incontinência anal pós-operatória e conseqüentemente, se for indicada esfincterotomia deve ser distal e bem limitada. A avaliação da extensão da ELI (feita pelos cirurgiões), correlacionada com o desenvolvimento de incontinência pós-operatória, sugere que esta incontinência possa ser causada por diminuição do segmento funcional do canal anal ${ }^{43}$. Entretanto, nos pacientes do presente estudo, submetidos à hemorroidectomia com ELI, apesar da zona de maior pressão ter diminuído mais acentuadamente nos pacientes incontinentes que nos continentes, essa diferença não foi estatisticamente significante.

Defeitos do esfíncter anal, porém assintomáticos, são comuns após partos vaginais, assim como defeitos do esfíncter externo, clinicamente silenciosos, presentes antes da ELI, tem sido implicados na gênese da incontinência fecal após a cirurgia. Deformidade anal também é considerada como responsável pelo desenvolvimento desta incontinência após esfincterotomia.

Apesar de a ELI promover menor grau de deformidade anal que a esfincterotomia posterior, ainda assim pode contribuir para a incontinência. Além disso, uma ELI mais extensa, por causar maior deformidade anal, pode resultar em incontinência ${ }^{43,44,45}$.

\section{CONCLUSÕES}

Hipertonia esfincteriana é comum em pacientes com doença hemorroidária avançada e parece exercer algum papel na sua formação. 
Rev bras Coloproct Julho/Setembro, 2007
Esfincterotomia lateral interna associada à hemorroidectomia no tratamento da doença hemorroidária. Vantagem ou desvantagem? Hélio Moreira Junior e Cols.
A manometria anorretal não identificou nenhum parâmetro pré-operatório que predissesse o risco de aparecimento de incontinência fecal no pós-operatório naqueles indivíduos submetidos à esfincterotomia lateral interna associada à hemorroidectomia.
Hemorroidectomia isolada não alterou, no pós-operatório, as pressões anais que eram elevadas no pré-operatório.

A esfincterotomia lateral interna, associada à hemorroidectomia, para o tratamento da doença hemorroidária avançada, além de não ter reduzido a dor pós-operatória, aumentou o risco de incontinência anal.

ABSTRACT: Background: The importance of using associated sphincterotomy for better pain control in patients who underwent hemorrhoidectomy remains controversial in the literature. Aim: Determine the role of associated sphincterotomy in patients submitted to surgical treatment for hemorrhoids. Patients and Methods: Twenty patients who underwent Milligan Morgan hemorrhoidectomy were distributed in two groups: Group 1: Hemorrhoidectomy without sphincterotomy and Group 2: Hemorrhoidectomy with sphincterotomy. Post-operative pain, complications as well anal continence was evaluated. Moreover, pre and post-operative manometry was performed, and collected data was compared between the two groups of patients. Results: There was no difference in the incidence of post-operative complications. Although group I used more narcotics and analgesics in the post-operative time, pain was significantly higher at $3^{\text {rd }}$ and $7^{\text {th }}$ post-operative day for Group II patients. Wound healing time was similar for both groups. Anal incontinence was significantly higher for patients who underwent sphincterotomy. Conclusion: Hemorrhoidectomy with associated internal lateral sphincterotomy did not reduce post-operative pain, and increased the risk of post-operative incontinence.

Key words: Hemorrhoids, Hemorrhoidectomy, Sphincterotomy, and Manometry.

\section{REFERÊNCIAS}

1. Thomson WHF. The nature of hemorrhoids. Br J Surg 1975; 62: 542-52.

2. Haas PA, Fox TA, Haas GP. The pathogenesis of hemorrhoids. Dis Colon Rectum 1984; 27: 442-50.

3. Wexner SD, Marchetti F, Jugelman DG. The role of sphincteroplasty for fecal incontinence re-evaluated: a prospective physiologic and functional review. Dis Colon Rectum 1991; 34: 22-30.

4. Schouten WR, Vroonhoven TJ. Lateral internal sphincterotomy in the treatment of hemorrhoids: A clinical and manometric study. Dis Colon Rectum1986; 29: 869-72.

5. Lin JK. Anal manometric studies in hemorrhoids and anal fissure. Dis Colon Rectum 1989; 32: 839-42.

6. Gorfine SR. Treatment of benign anal disease with topical nitroglicerin. Dis Colon Rectum 1995; 38: 453-7.

7. Farouk R, Duthie G, MacGregor A, Bartolo D. Sustained internal sphincter hypertonia in patients with chronic anal fissure. Dis Colon Rectum 1994; 37: 424-9.

8. Garcia-Aguilar J, Montes CB, Perez JJ, Jensen L, Madoff $\mathrm{RD}$, Wong WD. Incontinence after lateral internal sphincterotomy: anatomic and functional evaluation. Dis Colon Rectum 1998; 41: 423-7.

9. Wasvary HJ, Hain J, Mosed-Vogel M, Bendick P, Barkel DC, Klein SN. Randomized, prospective, double-blind, placebocontrolled trial of effect of nitroglycerin ointment on pain after hemorrhoidectomy. Dis Colon Rectum 2001; 44: 106973.
10. Lund JN, Scholefield JH. A randomized, prospective, doubleblind, placebo-controlled trial of glyceryl trinitrate ointment in treatment of anal fissure. Lancet 1997; 349: 11-4.

11. Corman ML. Hemorrhoids. Colon and Rectal Surgery. $3^{\text {rd }}$ ed. Philadelphia: JB Lippincott Company 1993. Chapter 2: 54115.

12. Galizia G, Lieto E, Castellano P, Pelosio L, Imperatore V, Pigantelli C. Lareral internal sphincterotomy together with haemorrhoidectomy for treatment of haemorrhoids: a randomized prospective study. Eur J Surg 2000; 166(3): 223-8.

13. Di Bella F, Estienne G. Indications for internal, medioposterior with anoplasty or lateral sphincterotomy during hemorrhoidectomy. Our experience. Minerva Chir 1990; 45(6): 361-3.

14. Khubchandani IT, Reed JF. Sequelae of internal sphincterotomy for chronic fissure in ano. Br J Surg 1989; 76: 431-4.

15. Garcia-Aguilar J, Belmonte C, Wong WD, Lowry AC, Madoff RD. Open vs. closed sphincterotomy for chronic anal fissure: long term results. Dis Colon Rectum 1996; 39: 440-3.

16. Rockwood TH,Church JM, Fleshman JW, Kane RL, Mavrantonis C, Thorson AG, Wexner SD, Bliss D, Lowry AC. Fecal incontinence quality of life scale: quality of life instrument for patients with fecal incontinence. Dis Colon Rectum 2000; 43: 9-17.

17. Kuypers JHC. Anal manometry, its applications and indications. Neth J Surg 1982; 34: 153-8.

18. Moreira Jr H , Wexner SD. Anorectal Physiologic Testing. In: Beck DE, Wexner SD (eds)Fundamentals of Anorectal Surgery. London: WB Saunders 1998:37-53. 
19. Gowers WR. The automatic action of the sphincter ani. Proc R Soc Lond 1877; 26: 77-84.

20. Bleday R, Pena JP, Rothenberger DA. Symptomatic hemorrhoids: current incidence and complications of operative therapy. Dis Colon Rectum 1992; 35: 477-81.

21. Johanson JF. Nonsurgical treatment of hemorrhoids. J Gastrointest Surg 2002; 6(3): 290-4.

22. Johanson JF, Rimm A. Optimal nonsurgical treatment of hemorrhoids: a comparative analysis of infrared coagulation, rubber band ligation, and injection sclerotherapy. Am J Gastroenterol 1992; 87: 1600-6.

23. MacRae HM, McLeod RS. Comparison of hemorrhoidal treatment modalities. A meta-analysis. Dis Colon Rectum 1995; 38: 687-94.

24. Milligan ET, Morgan CN, Lond LE. Surgical anatomy of the anal canal, and the operative treatment of hemorrhoids. Lancet 1937; 2: 1119-24.

25. Ferguson JA, Heaton JR. Closed hemorrhoidectomy. Dis Colon Rectum 1959; 2: 176-9.

26. Longo A. Treatment of haemorrhoidal disease by reduction of mucosa and haemorrhoidal prolapse with a circular-stapling device: a new procedure. Proceedings of $6^{\text {th }}$ World Congress of Endoscopic Surgery 1998; 777-84.

27. Helton WS. 2001 Consensus statement on benign anorectal disease. J Gastointest Surg 2002; 6(3): 302-3.

28. Senagore AJ. Surgical management of hemorrhoids. J Gastrointest Surg 2002; 6(3): 295-8.

29. Carapeti E, Kamm M, McDonald P, Phillips R. Double-blind randomized controlled trial of effects of metronidazole on pain after day-case haemorrhoidectomy. Lancet 1998; 351: 169-72.

30. Sobrado CW, Nahas SC, Marques CF, Habr-Gama A. Cirurgia ambulatorial em proctologia: experiência e análise do resultado de 503 operações. Rev Bras Coloproct 2001; 21(4): 22833.

31. Teramoto T, Parks AG, Swash M. Hypertrophy of the external anal sphincter in hemorrhoids: a histometric study. Gut 1981; 22: 45-8.

32. Sun WM, Peck RJ, Shorthouse AJ. Hemorrhoids are associated not with hypertrophy of the internal anal sphincter, but with hypertension of the anal cushion. Br J Surg 1992; 79: 592-4.

33. Segre D. Etiopathogenesis and physiopathology of hemorrhoidal disease. Ann Ital Chir 1995; 66: 747-50.
34. Hancock BD. Internal sphincter and the nature of the hemorrhoids. Gut 1997; 18: 651-5.

35. Mathai V, Ong BC, Ho YH. Randomized controlled trial of lateral internal sphincterotomy with haemorrhoidectomy. $\mathrm{Br}$ J Surg 1996; 83(3): 380-2.

36. Khubchandani IT. Internal sphincterotomy with hemorrhoidectomy does not relieve pain: a prospective, randomized study. Dis Colon Rectum 2002; 45: 1452-7.

37. Ho YH, Tan M. Ambulatory anorectal manometric findings in patients before and after haemorrhoidectomy. Int J Colorectal Dis 1997; 12: 296-7.

38. Chen WS, Leu SY, Wang FM. The roles of hemorrhoidectomy and lateral internal sphincterotomy in the treatment of hemorrhoids. Clinical and manometric study. Chung Hua I Hsueh Tsa Chih 1989; 43: 255-60.

39. Roe AM, Alexander JI. Pain scores in haemorrhoidectomy. $\mathrm{Br}$ J Surg 1988; 75: 293-4.

40. Ho YH, Seow-Choen F, Low JY, Tan M, Leong AP. Randomized controlled trial of trimebutine (anal sphincter relaxant) for pain after haemorrhoidectomy. Br J Surg 1997; 84: 377-9.

41. Lyngdorf P, Frimodt-Moller C, Jeppesen N. Voiding disturbances following anal surgery. Urol Int 1986; 41: 67-9.

42. Leong AF, Husain MJ, Seow-Choen F, Goh HS. Performing internal sphincterotomy with other anorectal procedures. Dis Colon Rectum 1994; 37: 1130-2.

43. Hananel N, Gordon PH. Lateral internal sphincterotomy for fissure-in-ano revisited. Dis Colon Rectum 1997; 40: 597-602.

44. Champigneule B, Dieterling P, Bigard MA, Gaucher P. Etude prospective de la fonction sphinctérienne anale avant et aprés hemorrhoidectomie. Gastroentrol Clin Biol 1989; 13: 452-6.

45. Chaves, DNB.; Petroianu A; Oliveira, HM. de; Capobiango, A.. Avaliação pós-operatória tardia de pacientes submetidos a esfincterotomia anal interna. Rev. Bras. Coloproctologia 20(3): 168-70, jul 2000

46. Brener, S; Souza, FJ, de; Matias, JEF. Resultado tardio da hemorroidectomia pela técnica de Milligan e Morgan. Rev. Bras. Coloproctologia 9(1): 12-5, jan.mar, 1989

\section{Endereço para correspondência:} PROF. HÉLIO MOREIRA

Fac. Medicina-U.F.Goiás-Depto.Cirurgia

Serviço de Coloproctologia. 